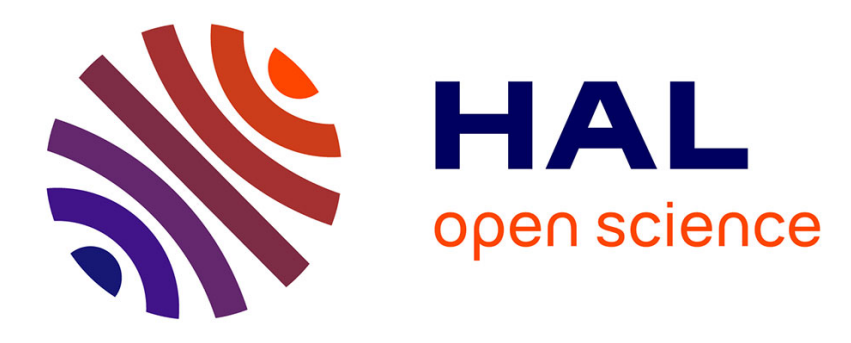

\title{
Balance émotionnelle dans les troubles anxieux et dépressifs
}

\author{
A. Pelissolo
}

\section{To cite this version:}

A. Pelissolo. Balance émotionnelle dans les troubles anxieux et dépressifs. Annales MédicoPsychologiques, Revue Psychiatrique, 2011, 169 (2), pp.124. 10.1016/j.amp.2010.12.005 . hal00727595

\section{HAL Id: hal-00727595 \\ https://hal.science/hal-00727595}

Submitted on 4 Sep 2012

HAL is a multi-disciplinary open access archive for the deposit and dissemination of scientific research documents, whether they are published or not. The documents may come from teaching and research institutions in France or abroad, or from public or private research centers.
L'archive ouverte pluridisciplinaire HAL, est destinée au dépôt et à la diffusion de documents scientifiques de niveau recherche, publiés ou non, émanant des établissements d'enseignement et de recherche français ou étrangers, des laboratoires publics ou privés. 


\section{Accepted Manuscript}

Title: Balance émotionnelle dans les troubles anxieux et dépressifs

Author: A. Pelissolo

PII: $\quad$ S0003-4487(10)00411-7

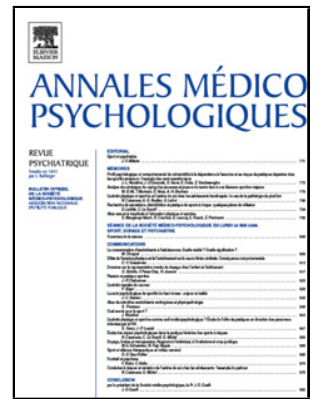

DOI: $\quad$ doi:10.1016/j.amp.2010.12.005

Reference: $\quad$ AMEPSY 1283

To appear in: $\quad$ Annales Médico-Psychologiques

Please cite this article as: Pelissolo A, Balance émotionnelle dans les troubles anxieux et dépressifs, Annales medio-psychologiques (2010), doi:10.1016/j.amp.2010.12.005

This is a PDF file of an unedited manuscript that has been accepted for publication. As a service to our customers we are providing this early version of the manuscript. The manuscript will undergo copyediting, typesetting, and review of the resulting proof before it is published in its final form. Please note that during the production process errors may be discovered which could affect the content, and all legal disclaimers that apply to the journal pertain. 


\title{
Communication
}

\section{Balance émotionnelle dans les troubles anxieux et dépressifs Emotional balance in anxiety and depressive disorders}

\author{
A. Pelissolo \\ Service de psychiatrie adulte et CNRS USR 3246, Hôpital Pitié-Salpêtrière (AP-HP), et \\ UPMC, 47, bd de l'Hôpital, 75013 Paris, France
}

Auteur correspondant: A. Pelissolo, Service de psychiatrie adulte et CNRS USR 3246, Hôpital Pitié-Salpêtrière (AP-HP), et UPMC, 47, bd de l'Hôpital, 75013 Paris, France Adresse email : antoine.pelissolo@upmc.fr

\section{Résumé}

Peu de travaux ont appliqué jusqu'à présent l'approche émotionnelle du modèle tripartite de Watson et Clark, différenciant affects positifs et négatifs, à l'étude des troubles anxieux et dépressifs. La notion de balance émotionnelle est pourtant intéressante pour la compréhension de ces pathologies, les deux dimensions pouvant évoluer différemment en fonction des syndromes cliniques. Notre étude porte sur un grand échantillon de patients souffrant de troubles dépressifs et anxieux, chez qui l'échelle EPN-31 (Émotionnalité Positive et Négative à 31 items) a été utilisée. Les résultats montrent essentiellement des profils émotionnels conformes au modèle tripartite : scores élevés d'affects négatifs dans les deux catégories de troubles, et scores faibles d'affects positifs surtout dans les troubles dépressifs. On trouve également des différences entre les troubles anxieux, avec en particulier des scores peu élevés d'affects négatifs dans le trouble panique et l'agoraphobie que dans les autres troubles anxieux. Ces résultats confirment la pertinence de cette approche émotionnelle, notamment pour des études longitudinales et des corrélations neurophysiologiques.

Mots clés : Affects négatifs ; Affects positifs ; Anxiété ; Dépression

\begin{abstract}
To date, few researches applied the emotional approach of the tripartite model of Watson and Clark, differentiating positive and negative affects, to the study of anxiety and depressive disorders. Nevertheless, the notion of emotional balance is interesting for the understanding of these pathologies and for the follow-up of treatments effects, both
\end{abstract}


dimensions being independent and able to evolve differently according to the clinical syndromes. In our study, a large sample of out-patients with depressive and anxiety disorders was explored, with the EPN-31 (Positive and Negative Emotionality) scale, and with other measures of anxiety and depressive symptoms. The results show were in line with the tripartite model of emotions: high scores of negative affects in both categories of disorders, and low scores of positive affects especially in the depressive disorders. These results confirmed the relevance of an emotional approach of anxiety and depressive disorders, in particular for longitudinal studies and neuro-physiological correlations.

Keywords: Anxiety; Depression; Negative affects; Positive affects

\section{Introduction}

La recherche actuelle en psychiatrie et en neurosciences est riche en travaux portant sur la régulation des émotions, dans des perspectives aussi bien physiopathologiques que thérapeutiques. Les outils sont de plus en plus sophistiqués pour explorer par exemple l'activité cérébrale accompagnant les réactions émotionnelles élémentaires ou plus complexes [4]. Cependant, relativement peu de recherches cliniques portent sur les processus émotionnels globaux, que l'on pourrait appeler des macro-affects, et leurs caractéristiques dans les troubles psychiatriques. Peu d'études se donnent pour objectif d'explorer, d'un point de vue clinique, les perturbations émotionnelles dans les troubles anxieux et dépressifs. Ces pathologies sont pourtant clairement des troubles dits «affectifs », et il serait essentiel de pouvoir mieux caractériser leurs dimensions émotionnelles propres. Pour pouvoir développer de tels travaux, le recours à des instruments de mesure fiables et précis des réactions émotionnelles est nécessaire, et il en existe aujourd'hui plusieurs dont l'utilisation est relativement simple en pratique courante, sous forme de questionnaires $[9,13]$. Notre objectif est donc d'appliquer ces approches psychométriques des émotions aux troubles anxieux et dépressifs, tout d'abord pour connaître leur validité et leur intérêt potentiel dans ces pathologies. Il s'agit ainsi d'un travail descriptif, pouvant servir de base à des recherches ultérieures centrées sur des objectifs physiopathologiques ou thérapeutiques spécifiques.

\section{La balance émotionnelle}


La notion de balance émotionnelle est directement issue des modèles psychologiques basés sur la distinction des émotions positives et négatives. Watson et Clark [1,2,10-13] ont notamment développé un modèle dit « tripartite » des émotions appliqué aux troubles anxieux et dépressifs. Selon cette approche, les troubles dépressifs se caractérisent par un niveau d'émotions positives anormalement bas et un niveau d'émotions négatives excessif, alors que les troubles anxieux sont également associés à des émotions négatives élevées, mais sans perturbation des émotions positives. La troisième composante de ce modèle décrit une dimension d'activation physiologique (neurovégétative) élevée dans l'anxiété et pas dans la dépression.

L'intérêt majeur de cette approche est que les deux catégories de macro-affects, positifs et négatifs, peuvent être mises assez directement en relation avec, respectivement, les systèmes cérébraux d'activation comportementale du plaisir et du renforcement (voies dopaminergiques notamment dans les noyaux accumbens), et les systèmes d'inhibition et de sécurisation (voies sérotoninergiques dans les régions limbiques en particulier) [4]. Au plan thérapeutique par ailleurs, il est maintenant classique de considérer que les traitements antidépresseurs inhibiteurs de recapture de la sérotonine ont pour principale cible émotionnelle les affects négatifs, que ce soit dans la dépression ou dans l'anxiété [6].

L'étude qui suit a donc consisté à mesurer, à l'aide d'un questionnaire, les affects positifs et négatifs de patients consultant pour un trouble affectif, et à comparer la balance émotionnelle associée aux différentes pathologies représentées dans cette population.

\section{Sujets et méthodes}

Les sujets inclus sont des patients reçus dans le service de psychiatrie de l'hôpital Pitié-Salpêtrière. Le critère d'inclusion principal est l'existence d'un trouble anxieux ou dépressif. Au cours d'une évaluation psychiatrique et psychométrique approfondie, les éléments suivants ont été pris en compte ici pour nos analyses : les diagnostics DSM-IV explorés à l'aide du Mini International Neuropsychiatric Interview (MINI) [8], et les scores aux échelles Émotionnalité Positive et Négative (EPN-31) [9], et Hospital Anxious and Depression scale (HAD) [14].

Les mesures émotionnelles ont donc été effectuées à l'aide de l'EPN-31, questionnaire créé initialement par E. Diener [5], adapté et traduit en Français par J.-P. Rolland et validé en population psychiatrique par Pélissolo et al. [9]. L'étude de validation de cet outil a confirmé notamment sa bonne structure factorielle et la bonne consistance interne des deux scores 
principaux [9]. Il n'existe pas à l'heure actuelle de valeur normative de l'EPN-31 en population générale, donc nous citerons comme seules valeurs de références disponibles les scores moyens obtenus dans une étude en cours dans un groupe de 100 étudiantes (âge moyen 26,3 ans ; écart-type 9,2) : affects positifs $70,1 \pm 16,0$ et affects négatifs $32,0 \pm 14,3$.

\section{Résultats}

\section{1. Échantillon}

La population étudiée est composée de 1215 patients, âgés de 37,5 ans $( \pm 13,3)$ en moyenne, avec une légère majorité de femmes $(53,3 \%)$. Un trouble de l'humeur, actuel ou passé, a été diagnostiqué chez $65,2 \%$ des patients, et au moins un trouble anxieux chez $81,3 \%$. Les troubles anxieux les plus fréquents dans cet échantillon sont les phobies sociales $(42,7 \%)$, les troubles obsessionnels compulsifs (TOC) $(22,6 \%)$, et le trouble panique et/ou l'agoraphobie (TPA) (21,6\%). Les scores moyens à l'échelle HAD sont 11,8 $\pm 4,4$ pour l'anxiété, et 8,0 $\pm 4,7$ pour la dépression.

\subsection{Scores émotionnels}

Les scores moyens obtenus à l'EPN-31 dans l'ensemble de l'échantillon sont les suivants (chacun pouvant varier entre zéro et 100) :

- chez les hommes $(n=567)$ : 47,9 pour les affects positifs, et 49,3 pour les affects négatifs ;

- chez les femmes $(n=648)$ : 52,7 pour les affects positifs, et 49,1 pour les affects négatifs.

Il existe une différence significative entre hommes et femmes uniquement pour le score d'affects positifs $(\mathrm{p}<0,001)$. Les deux scores émotionnels ne sont pas corrélés entre $\operatorname{eux}(r=-0,06)$.

\subsection{Comparaisons entre diagnostics}

En isolant tout d'abord les patients ayant un diagnostic DSM-IV de trouble dépressif actuel ou passé $(n=759)$ par rapport aux autres patients $(n=423)$, on obtient des scores significativement plus faibles d'affects positifs $(46,5$ vs 57,$1 ; \mathrm{p}<0,001)$, et plus élevés 
d'affects négatifs (53,8 vs 41,1; p < 0,001). La même comparaison concernant l'existence d'un trouble anxieux montre des tendances similaires mais statistiquement non significatives.

Le tableau I présente les moyennes obtenues chez des patients présentant des diagnostics de troubles anxieux «purs », c'est-à-dire un seul trouble parmi les cinq pris en compte. Ils sont comparés également aux patients ne présentant aucun trouble anxieux $(n=220)$. Globalement, l'analyse de variance ANOVA montre un effet significatif du diagnostic sur les scores d'affects positifs $(F=2,23 ; \mathrm{p}=0,049)$, et d'affects négatifs $(\mathrm{F}=9,86 ; \mathrm{p}<0,001)$. Cependant, aucun des tests post-hoc de comparaison des moyennes d'émotions positives entre les différents diagnostics pris deux à deux ne montre de différence significative. On note, en revanche, des différences significatives entre les scores d'émotions négatives des patients souffrant de TPA, d'une part, et les patients souffrant de phobie sociale ( $\mathrm{p}<0,001)$ ou de TOC ( $<0,001)$, d'autre part. Le TPA est donc caractérisé par des affects négatifs nettement moins élevés que dans les autres troubles anxieux, avec une moyenne se rapprochant de celle obtenue dans une population non clinique (38,2 vs 32$)$.

$<$ ici tableau I $>$

\subsection{Corrélations avec la HAD et le TCI-R}

Le tableau II rapporte les corrélations bivariées obtenues entre les scores de l'EPN-31, d'une part, et les échelles HAD et TCI-R, d'autre part. On y voit notamment que le score d'affects positifs est corrélé négativement aux scores d'anxiété $(\mathrm{r}=-0,18)$, mais surtout de dépression $(r=-0,50)$, alors que le score d'affects négatifs est corrélé positivement presque autant aux scores de dépression $(\mathrm{r}=0,5)$ que d'anxiété $(\mathrm{r}=0,61)$. Une analyse de régression linéaire, considérant les scores de l'EPN-31 séparément comme variables dépendantes, et les scores HAS anxiété et dépression, ainsi que l'âge et le sexe des patients comme variables dépendantes, confirme que les affects positifs sont surtout déterminés par le niveau de dépression ( $\mathrm{t}=-18,0 ; \mathrm{p}<0,001)$, et peu par le niveau d'anxiété $(\mathrm{t}=2,4 ; \mathrm{p}=0,02)$, alors que les affects négatifs sont déterminés autant par le niveau de dépression $(\mathrm{t}=19,0 ; \mathrm{p}<0,001)$ que d'anxiété $(\mathrm{t}=11,4 ; \mathrm{p}<0,001)$.

$<$ ici tableau $2>$

\section{Discussion et conclusion}


Ces premières données explorant la balance émotionnelle entre affects positifs et négatifs à l'aide de l'EPN-31 dans un très grand échantillon de patients souffrant de troubles dépressifs et anxieux montrent l'intérêt d'une telle approche. En effet, l'existence de différences assez nettes en fonction des diagnostics est un résultat nouveau, même si quelques études antérieures, menées avec d'autres instruments et dans des populations plus réduites, avaient pu suggérer les mêmes tendances $[2,12,13]$. Les scores assez faibles d'affects négatifs retrouvés chez les patients souffrant de troubles paniques ou d'agoraphobie par rapport aux autres troubles anxieux permettent d'envisager des hypothèses sur les mécanismes de régulation émotionnelle (ou d'évitement) chez ces patients, limitant ainsi la fréquence des émotions négatives. En revanche, l'absence de différence nette d'un trouble anxieux à l'autre concernant les affects négatifs est relativement surprenante pour les phobies sociales, car plusieurs études antérieures ont suggéré l'existence d'un émoussement des émotions positives dans cette pathologie, même en l'absence de trouble dépressif [7].

Par ailleurs, nos résultats confirment les hypothèses du modèle tripartite des émotions, les affects positifs (bas) étant surtout associés à la dépression, alors que les affects négatifs se retrouvent élevés aussi bien dans l'anxiété que dans la dépression [10].

Les principales limites de notre étude sont l'absence de mesure objective des réactions émotionnelles chez les patients inclus, la spécificité de la population hospitalière prise en compte, et la nature uniquement transversale de l'évaluation, alors que certains patients sont sous traitement psychotropes notamment. Les études à venir devront donc tenir compte de ces limites, en tentant de croiser les évaluations de 1'EPN-31 avec des mesures objectives de réactivité émotionnelle ou d'autres explorations neurophysiologiques, et en procédant à des recueils longitudinaux permettant d'observer les évolutions des profils émotionnels dans le temps, en particulier sous traitement. 
Tableau I : Scores de l'EPN-31 en fonction des diagnostics de troubles anxieux « purs » (patients ne présentant qu'un seul des cinq troubles anxieux pris en compte)

\begin{tabular}{llllll}
\hline & \multicolumn{3}{c}{ Affects positifs } & \multicolumn{2}{c}{ Affects négatifs } \\
& $\mathrm{N}$ & Moyenne & Écart-type & Moyenne & Écart-type \\
\hline Phobie sociale & 330 & 48,1 & 21,6 & 51,2 & 19,4 \\
Trouble anxieux généralisé & 53 & 52,1 & 20,1 & 49,5 & 20,7 \\
Trouble panique et/ou agoraphobie & 127 & 53,5 & 18,9 & 38,2 & 18,9 \\
Trouble obsessionnel-compulsif & 148 & 50,6 & 21,2 & 54,0 & 20,7 \\
État de stress post-traumatique & 22 & 42,5 & 25,6 & 44,0 & 28,6 \\
\hline Aucun trouble anxieux & 220 & 52,1 & 22,9 & 47,0 & 23,0 \\
\hline Total & 900 & 50,3 & 21,6 & 48,5 & 21,3 \\
\hline
\end{tabular}

Tableau II. Corrélations bivariées (r de Pearson) entre les scores de l'EPN-31 et les scores aux échelles HAD et TCI-R sur l'ensemble de l'échantillon (n=1215)

\begin{tabular}{llcccc}
\hline & \multicolumn{2}{c}{ Affects positifs } & \multicolumn{2}{c}{ Affects négatifs } \\
& & $\mathrm{r}$ & $\mathrm{p}$ & $\mathrm{r}$ & $\mathrm{p}$ \\
\hline HAD & Anxiété & $-0,18$ & $<0,001$ & 0,61 & $<0,001$ \\
& Dépression & $-0,50$ & $<0,001$ & 0,50 & $<0,001$ \\
& & & & \\
TCI-R & Recherche de nouveauté (NS) & 0,21 & $<0,001$ & $-0,04$ & 0,14 \\
& Évitement du danger (HA) & $-0,31$ & $<0,001$ & 0,45 & $<0,001$ \\
& Dépendance à la récompense (RD) & 0,34 & $<0,001$ & $-0,1$ & $<0,001$ \\
& Persistance (P) & 0,25 & $<0,001$ & $-0,11$ & $<0,001$ \\
& Détermination (SD) & 0,32 & $<0,001$ & $-0,52$ & $<0,001$ \\
& Coopération (C) & $-0,20$ & $<0,001$ & $-0,20$ & $<0,001$ \\
& Transcendance (ST) & 0,17 & $<0,001$ & 0,11 & $<0,001$ \\
\hline
\end{tabular}

\section{Références}

[1] Clark LA, Watson D. Tripartite model of anxiety and depression: psychometric evidence and taxonomic implications. J Abnormal Psychol 1991;100:316-36.

[2] Clark LA, Watson D, Mineka S. Temperament, personality, and the mood and anxiety disorders. J Abnormal Psychol 1994;103:103-16. 
[3] Cloninger CR, Svrakic DM, Przybeck TR. A psychobiological model of temperament and character. Arch Gen Psychiatry 1993;50:975-90.

[4] Davidson RJ. Anxiety, depression, and emotion. New York: Oxford University Press; 2000 .

[5] Diener E, Smith H, Fujita F. The personality structure of affect. J Personal Soc Psychol 1995;69:130-41.

[6] Harmer CJ, O’Sullivan U, Favaron E, et al. Effect of acute antidepressant administration on negative affective bias in depressed patients. Am J Psychiatry 2009;166:1178-84.

[7] Kashdan TB. Social anxiety spectrum and diminished positive experiences: theoretical synthesis and meta-analysis. Clin Psychol Rev 2007;27:348-65.

[8] Lecrubier Y, Sheehan DV, Weiller E, et al. The Mini International Neuropsychiatric Interview (MINI) a short diagnostic structured interview: reliability and validity according to the CIDI. Eur Psychiatry 1997;12:224-31.

[9] Pelissolo A, Rolland JP, Perez-Diaz F, et al. L'évaluation dimensionnelle des émotions en psychiatrie : validation du questionnaire Émotionnalité Positive et Négative à 31 items (EPN31). Encéphale 2007;33:256-63.

[10] Watson D, Clark LA, Carey G. Positive and negative affectivity and their relation to anxiety and depressive disorders. J Abnormal Psychol 1988; 97:346-53.

[11] Watson D, Clark LA, Weber K, et al. Testing a tripartite model: I. Evaluating the convergent and discriminant validity of anxiety and depression symptom scales. $\mathbf{J}$ Abnormal Psychol 1995;104:3-14.

[12] Watson D, Clark LA, Weber K, Assenheimer JS, Strauss ME, Mc Cormick RA. Testing a tripartite model: II. Exploring the symptom structure of anxiety and depression in student, adult, and patient samples. J Abnormal Psychol 1995;104:15-25

[13] Watson D. Mood and temperament. New York: The Guilford Press; 2000.

[14] Zigmond AS, Snaith RP. The Hospital Anxiety and Depressive Scale. Acta Psychiatr Scand 1983;67:361-70.

\section{Discussion}

Mme L. Sala - Votre échelle est-elle utilisable aussi dans d'autres pathologies que celles que tu vous citez dans votre étude? Est-elle utilisable pour les états limites ?

Réponse du Rapporteur - Oui, tout à fait, car cette mesure dimensionnelle des affects positifs et négatifs peut s'appliquer à toutes les pathologies de l'axe 1 et de l'axe 2 dans 
lesquelles il existe des troubles émotionnels. En revanche, elle n'a pas été conçue (et aucune validation n’a été effectuée à ce jour) pour être utilisée chez des patients psychotiques.

$\operatorname{Dr}$ N. Tesu-Rollier - Ma question porte sur une corrélation partielle entre la catégorie clinique «intolérance à la frustration » et le dégoût de la catégorie «anxiété » de l'échelle EPN-31.

Réponse du Rapporteur - On peut envisager cette association mais nous ne l'avons pas recherché spécifiquement. La sous-dimension «dégoût» est assez spécifiquement et logiquement élevée chez les patients souffrant de troubles obsessionnels-compulsifs. 